\title{
Ambulatory Motor Assessment in Parkinson's Disease
}

\author{
Noël L.W. Keijsers, $\mathrm{PhD},{ }^{1 *}$ Martin W.I.M. Horstink, $\mathrm{MD}, \mathrm{PhD},{ }^{2}$ and Stan C.A.M. Gielen, $\mathrm{PhD}^{1}$ \\ ${ }^{I}$ Department of Biophysics, Institute for Neuroscience, Radboud University Nijmegen, Nijmegen, The Netherlands \\ ${ }^{2}$ Department of Neurology, Institute for Neuroscience, University Medical Center St. Radboud, Nijmegen, The Netherlands
}

\begin{abstract}
We developed an algorithm that distinguishes between on and off states in patients with Parkinson's disease during daily life activities. Twenty-three patients were monitored continuously in a home-like situation for approximately 3 hours while they carried out normal daily-life activities. Behavior and comments of patients during the experiment were used to determine the on and off periods by a trained observer. Behavior of the patients was measured using triaxial accelerometers, which were placed at six different positions on the body. Parameters related to hypokinesia (percentage movement), bradykinesia (mean velocity), and tremor (percentage peak frequencies above $4 \mathrm{~Hz}$ ) were used to distinguish between on and off states. The on-off detection was evaluated using sensitivity and specificity. The performance for each patient
\end{abstract}

was defined as the average of the sensitivity and specificity. The best performance to classify on and off states was obtained by analysis of movements in the frequency domain with a sensitivity of 0.97 and a specificity of 0.97 . We conclude that our algorithm can distinguish between on and off states with a sensitivity and specificity near 0.97 . This method, together with our previously published method to detect levodopa-induced dyskinesia, can automatically assess the motor state of Parkinson's disease patients and can operate successfully in unsupervised ambulatory conditions. (C) 2005 Movement Disorder Society

Key words: Parkinson's disease; motor fluctuations; automatic assessment; accelerometers; daily life
During the first years of levodopa (L-dopa) treatment, patients with Parkinson's disease (PD) have a stable response to L-dopa. After several years of L-dopa treatment, however, an increasing number of patients show fluctuations in motor response and L-dopa-induced dyskinesias (LID). ${ }^{1-4}$ These complications constitute a major problem in the long-term management of PD and add substantially to the patient's disability. Two main problems arise from a therapeutical point of view: first, the clinical state of patients has to be determined (on, off, or LID), and second, it has to be known how this clinical state fluctuates over time during the course of the day. Many methods have been developed to assess these late L-dopa problems in PD; however, the standard clinical detection and rating methods can only be applied in a hospital setting under supervision of a trained clinical

\footnotetext{
*Correspondence to: Dr. N.L.W. Keijsers, Dept. of Biophysics, Institute for Neuroscience, Radboud University Nijmegen, Geert Groote plein 21, 6525 EZ Nijmegen, Postbus 9101, The Netherlands. E-mail: n.keijsers@science.ru.nl

Received 7 December 2004; Revised 25 February 2005; Accepted 12 March 2005

Published online 25 August 2005 in Wiley InterScience (www. interscience.wiley.com). DOI: 10.1002/mds.20633
}

observer. ${ }^{5-7}$ Moreover, these rating methods provide only a momentary assessment of the clinical condition. This is not sufficient for practical purposes, because fluctuations over time require long-term supervision of up to a few days.

For long-term evaluation of PD symptoms, patients usually have to keep a diary to record whether they are on, have LID, or are off (reemergence of PD symptoms). However, self-report of the motor-state in diaries has several limitations and can be troublesome or even unreliable. ${ }^{8-10}$ For example, Goetz and colleagues ${ }^{11}$ tested the efficacy of a patient-training videotape on motor fluctuations for on-off diaries. They showed that the ratings by 12 of 32 PD patients differed by more than $80 \%$ from those of a trained observer. Therefore, automatic ambulatory assessment of the motor state and motor fluctuations with a high performance would be highly useful in the management of PD and in the evaluation of surgical and pharmacologic interventions.

As explained above the currently available standard clinical methods to assess the motor state in PD patients have several limitations and cannot be used in daily life. Activity monitors have been used mainly to identify the 
energy expenditure in daily life ${ }^{12,13}$ but have also been used to assess motor fluctuations in daily life. Studies that focused on the assessment of diurnal motor fluctuations in PD used a wrist-worn activity monitor. ${ }^{14-17}$ These studies measured the number of activities in a short interval ( 15 seconds to 5 minutes) and found a global relation between the number of activities and response fluctuations. The validity of this wrist-worn activity monitor in terms of sensitivity and specificity in detecting on and off was not determined. This is unfortunate because validation is a crucial step for a successful automatic detection of on and off states in daily life. A disadvantage of the wrist-worn activity monitor used in these studies is that this monitor only indicates the amount of movement and does not give a good representation of the functionality or nature of the movements. For example, the activity monitor could not distinguish between voluntary movements, LID, and tremor. The wrist-worn activity monitor therefore was only able to assess the duration of immobility periods as a measure of hypokinesia. Because PD patients in the off state can show various, interindividual differences in PD symptoms, an ambulatory device has to be able to assess all possible PD symptoms that are characteristic for the off state such as bradykinesia, tremor, and akinesia. More recently, multichannel accelerometry has been used in the detection and assessment of motor disorders in PD in daily life. ${ }^{18,19}$ In particular, we used multichannel accelerometry and a neural network approach with good success to assess the severity of dyskinesia and to distinguish dyskinesia from voluntary movements in daily life. ${ }^{20} \mathrm{An}$ advantage of this method is that movements of several segments can be measured simultaneously and that the accelerometer signals can be used to calculate several variables related to Parkinson symptoms. So far, only Hoff and colleagues ${ }^{21}$ have investigated the sensitivity and specificity of continuous ambulatory multichannel accelerometry in detecting on and off states while patients were at home. They used three different variables (immobility duration, mean acceleration, and percentage time with tremor) that were calculated for 30-minute intervals using the accelerometers on the wrist. The three variables were used separately to detect on and off states. Hoff and coworkers ${ }^{21}$ reported a specificity for hypokinesia, bradykinesia, and tremor in the range between 0.66 and 0.76 and a sensitivity in the range between 0.60 and 0.71 .

The relatively low values for the sensitivity and specificity found in the study by Hoff and associates ${ }^{21}$ could be the result of several factors. One of the possible factors suggested by the authors was the use of subjective self-assessment as a reference. The poor perfor- mance could be due to the poor validity of self-assessment and assessment of a trained observer might solve this problem. Another reason for the relatively small sensitivity and specificity might be that the variables to detect bradykinesia and hypokinesia were based on the accelerometer signals in the frequency range between 1 and $3.5 \mathrm{~Hz}$. Because many movements in daily life occur at frequencies below $1 \mathrm{~Hz},{ }^{22}$ much relevant information about daily life motor activity might have been lost by focusing on the $1-$ to $3.5-\mathrm{Hz}$ frequency range. A final reason for the low sensitivity and specificity is that PD patients show different interindividual PD symptoms in the off state. An algorithm that is based on more PDrelated parameters than the three variables used in the study by Hoff and colleagues ${ }^{21}$ might therefore lead to a more successful detection of on-off fluctuations for all PD patients.

The main purpose of this study is to develop an algorithm for the automatic detection of motor fluctuations in Parkinson's disease that gives a significantly higher sensitivity and specificity than that obtained in the study by Hoff and coworkers ${ }^{21}$ We will use variables related to bradykinesia, hypokinesia, and tremor to distinguish between on and off states. For bradykinesia, we will calculate the mean velocity over periods of 1 minute and the mean velocity during movements. The amount of time that a segment is moving is used as a variable for hypokinesia. A parkinsonian rest tremor has a typical frequency between 4 and $8 \mathrm{~Hz}$ but an action or postural tremor could also be present at higher frequencies; ${ }^{23,24}$ therefore, the percentage of peak frequencies above $4 \mathrm{~Hz}$ will be used as a variable related to tremor. In addition to single variables, more sophisticated classification techniques, that combine several variables, will be used to detect on and off states.

\section{PATIENTS AND METHODS}

\section{Patients}

Twenty-three patients (17 men, 6 women) with on-off fluctuations participated in this study. Patients had either end-of-dose or random off periods. All patients were known to us for many years and could accurately describe relevant states and symptoms. Patients were not demented and did not suffer from depression. None of the patients had nonmotor side effects of therapy. In the on state, patients were in Hoehn-Yahr stage II to III, and in the off state usually in Hoehn-Yahr stage III to IV. Occasionally, some patients were unable to walk if they had problems with L-dopa absorption or when they did not take their L-dopa dose in time. 
The experiments were approved by the Medical Ethical Committee of the University Medical Center of the University of Nijmegen. All patients gave informed consent. Patients were between 39 and 71 years old (mean \pm standard deviation [SD], $57 \pm 8$ years) and had a mean duration of the disease of $14 \pm 4$ years (range, 7-23 years). Mean L-dopa medication was $695 \pm 300 \mathrm{mg}$ daily (range, 375-1,400 mg/day). In addition to L-dopa, 11 patients were on pergolide (mean $\pm \mathrm{SD}, 4.4 \pm 2.5$ ), 5 patients were on pramipexol $(0.82 \pm 0.65)$, and 1 patient was on ropinirol ( $2.75 \mathrm{mg} /$ day). Patients were monitored continuously for approximately 3 hours (mean [SD], 172 (30) minutes; range, 130-230 minutes). During this period, the patients took their regular medication at their usual time. When a patient did not switch between a state, however, L-dopa medication was postponed or an extra dose was taken to induce an off or on state, respectively. During the test, 17 patients showed fluctuations with both on and off periods, whereas 3 patients were only in an on period and 3 others were only in an off period. Of 20 patients who showed an off period, $11 \mathrm{did}$ not suffer from a tremor and 9 did. Each recording session started between $10 \mathrm{Am}$ and 1 PM. The experiments took place in a home-like setting in the occupational therapy department of the Radboud University Nijmegen Medical Center. During the monitoring session, the patients carried out approximately 35 functional daily life activities, such as putting on a coat, reading a newspaper, making a phone call, washing hands, and walking. We intended to imitate the behavior of the patients in normal daily life. Behavior in daily life is not constrained by standardized execution of defined tasks, but both the type and execution of free behavior differ throughout the day both within and between subjects. We therefore created an environment in which they could carry out daily life activities in a natural home-like setting. Above all, patients had to carry out the tasks in their own preferred way. At the start of the experiment, subjects were instructed to carry out all activities in their own way and at their own pace. The experimenter gave instructions about the order of activities (e.g., preparing lunch, reading the newspaper, making coffee, etc.), keeping them alert and active at all times. A large number of tasks were carried out in the off state as well as in the on state. Patients were free to take a rest between activities at any time. During these "rest" periods, patients mainly were sitting in a chair and chatting. The rest periods were always small and did not exceed 10 minutes.

\section{Data Acquisition}

The movements of the trunk, upper arms, and postures were measured automatically using accelerometers. Six

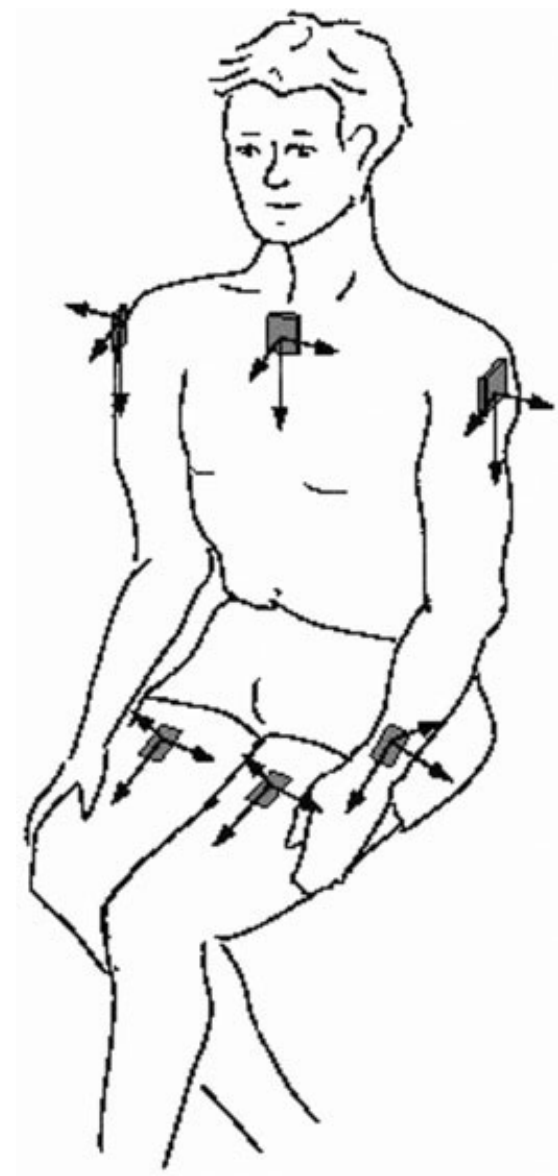

FIG. 1. Schematic overview of the position of the six sets of accelerometers on the body. The three orthogonal directions for measurement of acceleration by each set of accelerometers are indicated by arrows.

sets with three orthogonal accelerometers each (ADXL202; Analog Devices, Norwood, MA) were placed at six different positions on the body (Fig. 1): at both upper arms (just below the shoulder in the sagittal plane), at both upper legs (halfway the upper leg on the anterior side), at the wrist of the most affected side, and at the trunk (top of the sternum). The accelerometer signals were stored digitally on a data recorder (Vitaport 3; TEMEC Instruments, Kerkrade, The Netherlands) that was attached to a belt around the patient's waist. The accelerometer signals were sampled at a frequency of $256 \mathrm{~Hz}$, low-pass filtered using a moving averaging window with a $3 \mathrm{~dB}$ cut-off frequency at $16 \mathrm{~Hz}$ and stored at a sample frequency of $64 \mathrm{~Hz}$. During the whole experiment, patients were observed by the experimenter and their behavior was videotaped. The experimenter observed the behavior of the patient and asked the patient about his/her motor state, especially when he observed small changes in motor state. Patients also were in- 
structed to mention any change in motor state. Only patients who could clearly identify their motor state were included in this study. The experimenter used any sign of PD symptoms such as tremor, bradykinesia, and hypokinesia to determine whether a patient was in an off state. Patients were performing motor tasks every 10 minutes during which they were moving most of the time; therefore, any sign of bradykinesia could be observed clearly during the whole experiment. All periods, in which patients did not show any sign of PD symptoms and in which the patients classified their self as on, were classified as on state. Periods in which patients showed dyskinesia were also classified as on state. The clinical classification of on and off states was thus based on the observation of the experimenter together with the comments of the patient. If necessary, the videotapes were used to check the motor state and the kind of task carried out by a patient. It is often hard to detect the precise timing of a switch between motor states; therefore, we did not use the data in a window of 5 minutes around the time of a switch, indicated by the trained observer or patient, to develop our algorithm.

Of the 23 patients of this study, 13 had participated in our previous study to assess the severity of dyskinesia. In the previous study, only the data in the on state had been used and we had been able to distinguish between dyskinesias and voluntary movements. ${ }^{20}$ In the present study, we also used the data of the off states of the patients. In addition, we did not rate the severity of dyskinesia in the present study, on periods with and without dyskinesia were just classified as on state.

\section{Data Analysis}

Four parameters were calculated from the accelerometer signals to distinguish between the on and off state of PD patients. These variables were related to three of the core features of PD namely, bradykinesia (slowness of voluntary movement), hypokinesia (lack of voluntary movements), and tremor (spontaneous rhythmic repetitive alternating movements). Each parameter was calculated for each body segment for subsequent 1-minute intervals. An accelerometer signal consists of two different components, which cannot be distinguished: a component related to acceleration of the moving limb and a gravity component related to the position of the accelerometer relative to gravity. Any movement will affect both components and thus any change in the accelerometer signal will reflect movement of the accelerometer. For this reason, the derivative of the accelerometer signal was used as a measure for movement of the body segment. The square root of the sum of squares of the derivatives of the three orthogonal accelerometer signals from a body segment will be referred to as "segment velocity" for that limb segment. For assessment of bradykinesia and hypokinesia, three different variables were calculated. For the calculation of the bradykinesia and hypokinesia variables, the raw accelerometer signal was first filtered by a second-order low-pass digital Butterworth filter with a 3-dB cut-off frequency at $3.5 \mathrm{~Hz}$. The segment velocity was calculated thereafter using the filtered accelerometer signals. The variables $\overline{\mathrm{V}}$ and $\overline{\mathrm{V}}_{\theta}$ represent the mean velocity of a segment and the mean velocity when a segment moves, respectively. Movement of a segment was defined as the condition when the segment velocity exceeded a threshold of $0.05 \mathrm{~m} / \mathrm{sec}$.

For the assessment of hypokinesia, the percentage of time that a segment is moving was calculated $\left(\% \mathrm{~V}_{\theta}\right)$.

In addition to the bradykinesia and hypokinesia related variables, we calculated a variable to detect tremor. A parkinsonian rest tremor has a typical frequency between 4 and $8 \mathrm{~Hz}$ but an action or postural tremor could also be present at higher frequencies. ${ }^{23,24}$ The percentage of peak frequencies above $4 \mathrm{~Hz}$ (indicated by $\% \mathrm{PF}_{4}$ ) therefore was used as a variable to detect PD tremor. For the calculation of the percentage of peak frequencies above $4 \mathrm{~Hz}$, the following procedure was used. The peak frequency was calculated using a moving window of 3 seconds, sliding 1 second per step. For each 3 -second interval, the raw accelerometer signal was filtered with a high-pass digital filter with a 3-dB cut-off frequency at 1 $\mathrm{Hz}$ and subsequently multiplied by a Blackman window to align begin and end of the 3 -second window. Fourier analysis was applied on this signal to calculate the frequency spectrum. The sum of the amplitude spectra of the three accelerometers on each body segment was taken and the peak frequency was the frequency with the largest power. In daily life, walking is one of the activities in which the velocity of body segments is relatively large compared to that in other daily life activities. In both on and off states, our patients were able to walk for several minutes. Walking can be detected easily using accelerometers on both legs 25,26 and we excluded the data in which patients walked continuously for more than 3 minutes.

Patients may reveal small fluctuations of the variables, which might not necessarily reflect a switch between on and off states. The value of each of the five variables as a function of time therefore was filtered with a secondorder digital Butterworth filter with a cut-off frequency at $0.001 \mathrm{~Hz}$ to reveal slow trends (on a 15-minute time scale) of the on-off states.

Patients who suffer from bradykinesia and hypokinesia will make slow and fewer movements in the off state. For this reason, bradykinetic and hypokinetic patients 
were expected to show small values for the variables $\overline{\mathrm{V}}$, $\overline{\mathrm{V}}_{\theta}$, and $\% \mathrm{~V}_{\theta}$ in the off state. Patients with a tremor would most likely show large values for the variable $\% \mathrm{PF}_{4}$ in the off state. A threshold for each of these variables was computed to distinguish between on and off states, as will be explained below. The on-off detection was evaluated using the sensitivity and specificity, which were defined as the fraction of correctly classified data in the off and on state, respectively. For each variable, the sensitivity and specificity depend on the threshold for that variable, which discriminates between the on and the off state. Sensitivity and specificity were used to produce a receiver operating characteristics (ROC) curve. The optimal threshold to discriminate between on and off states was chosen as the threshold that gave the smallest distance of the ROC curve to the maximum (sensitivity and specificity of 1.00).

In addition to sensitivity and specificity, the performance for each patient was calculated as the average of the sensitivity and specificity ([sensitivity + specificity]/ 2). When a patient was only tested in the off (or on) state, the performance was equal to the correct classification in the off (= specificity) or on (= sensitivity) state.

In general, PD patients can be subdivided in akineticrigid-dominant patients and tremor-dominant patients. Patients were thus divided in a group who suffered from a visible tremor in the off state (tremor group) and a group who did not suffer from tremor (nontremor group). The selection was based on the observation of the experimenter and on the evaluation of the videotapes by a trained physician. To be classified as a patient with a tremor, patients had to show a visible tremor anywhere during the experimental session. Patients who did not show a visible tremor during the experiment were classified as nontremor patients. Nine patients showed a visible tremor and were selected for the tremor group. The remaining 13 patients (1 patient was left out, discussed below) were selected for the nontremor group.

In addition to the detection of on-off states based on each of the single variables, we explored whether a combination of variables could give a better performance in detecting on-off states. For each of the six body segments, all four variables were calculated resulting in a total of 24 variables. These variables were used as input to a neural network (multilayer perceptron [MLP]) with an input layer, one hidden layer, and an output layer. The output of the hidden units and the output unit was given by a hyperbolic tangent sigmoid transfer function that gives a value between -1 and +1 . The output of the unit in the output layer represents the on $(-1)$ or the off $(+1)$ state. The neural network was optimized using the mean square error (MSE) between the neural network output and the state indicated by the trained physician. For more detailed information about the neural networks, see Keijsers and colleagues. ${ }^{20}$

Neural networks with various numbers of hidden units were used and forward selection was used to find the most important input variables to distinguish between motor states. Forward selection means that we started with an empty set of variables and added, one after another, the variable that causes the largest reduction of the MSE between the neural network output and the score given by the physician. The neural network was seen as correct when the neural network gave a score larger than 0 for the off state and smaller than 0 for the on state.

In conclusion, four different variables $\left(\overline{\mathrm{V}}, \overline{\mathrm{V}}_{\theta}, \% \mathrm{~V}_{\theta}\right.$, and $\% \mathrm{PF}_{4}$ ) for each of the six body segments were determined. For each of the variables and for each segment, the threshold was optimized to achieve the largest sensitivity and specificity in detecting on and off states. In addition, neural networks were used to explore whether a combination of parameters could optimize the performance of detecting on and off states.

\section{RESULTS}

The main purpose of this study was to develop an algorithm that is able to distinguish between on and off states in daily life. Figure 2 shows the performance (average of sensitivity and specificity) of the variables of the wrist (upper panel), trunk (middle panel), and the leg (lower panel) for the two groups of patients and for the group as a whole. In our analysis we will refer to the wrist and the leg that are most affected by the PD symptoms. Table 1 shows the average and standard deviation of the sensitivity and specificity in detecting on and off states by each of the variables $\overline{\mathrm{V}}, \overline{\mathrm{V}}_{\theta}, \% \mathrm{~V}_{\theta}$, and $\% \mathrm{PF}_{4}$ for the different patient groups. Column 3 of Table 1 shows the body segment that gave the best performance for each variable and patient group. The performance is shown in Figure 2. Data of one patient was left out for reasons that are explained at the end of this section.

The variable $\% \mathrm{PF}_{4}$ of the trunk gave the best performance in the detection of on and off states for all PD patients (performance, $0.96 \pm 0.09$; sensitivity, $0.96 \pm$ 0.11 ; and specificity, $0.95 \pm 0.12$ ) and the nontremor group (performance, $0.96 \pm 0.09$; sensitivity, $0.97 \pm$ 0.08 ; and specificity, $0.96 \pm 0.06$ ). For the tremor group, the variable $\% \mathrm{PF}_{4}$ of the most affected leg gave the best performance (performance, $0.97 \pm 0.04$; sensitivity, $0.97 \pm 0.07$; and specificity, $0.97 \pm 0.05$ ). The variables $\overline{\mathrm{V}}, \overline{\mathrm{V}}_{\theta}$, and $\% \mathrm{~V}_{\theta}$ indicated that PD patients move slower and move less frequent in the off state than they do in the 


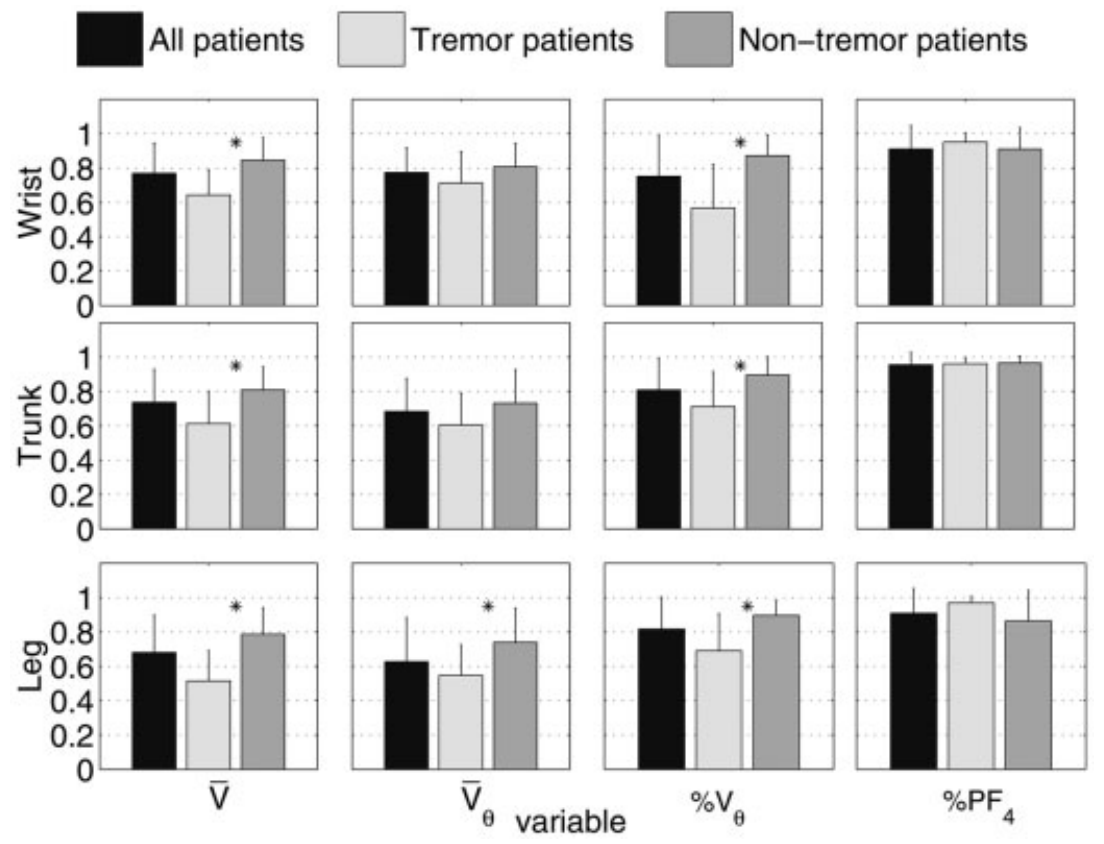

FIG. 2. The performance (average of sensitivity and specificity) of the variables for bradykinesia $\left(\overline{\mathrm{V}}\right.$ and $\left.\overline{\mathrm{V}}_{\theta}\right)$, hypokinesia $\left(\% \mathrm{~V}_{\theta}\right)$, and tremor (and $\% \mathrm{PF}_{4}$ ) of the wrist (upper panel), trunk (middle panel), and leg (lower panel) for all patients (dark bars), for patients with a tremor (white bars), and for patients without a tremor (gray bars). Error bars represent standard deviation. *Significant difference between the nontremor and tremor group.

on state but these variables showed smaller sensitivity and specificity values in detecting on and off states than did the variable $\% \mathrm{PF}_{4} \cdot \overline{\mathrm{V}}$ and $\% \mathrm{~V}_{\theta}$ gave a significantly better performance for the nontremor group than for the tremor group (unpaired $t$ test, $\overline{\mathrm{V}}$ and $\% \mathrm{~V}_{\theta}$ of the wrist: $P<0.005, \overline{\mathrm{V}}$ and $\% \mathrm{~V}_{\theta}$ of the trunk: $P<0.01$, and $\overline{\mathrm{V}}$ and $\% \mathrm{~V}_{\theta}$ of the most affected leg: $\left.P<0.005\right)$. The variable $\overline{\mathrm{V}}_{\theta}$ gave a significantly better performance for the non-

TABLE 1. Sensitivity and specificity in detecting on and off states by each of the variables

\begin{tabular}{llll}
\hline \multicolumn{1}{c}{ Patient group } & $\begin{array}{c}\text { Body } \\
\text { segment }\end{array}$ & Sensitivity & Specificity \\
\hline Mean segment velocity $(\bar{V})$ & & & \\
$\quad$ All patients & Wrist & $0.71(0.30)$ & $0.78(0.27)$ \\
$\quad$ Tremor & Wrist & $0.58(0.34)$ & $0.70(0.24)$ \\
$\quad$ Nontremor & Wrist & $0.81(0.30)$ & $0.86(0.17)$ \\
Mean segment velocity & & & \\
$\quad$ when moving $\left(\bar{V}_{\theta}\right)$ & & & \\
All patients & Wrist & $0.74(0.32)$ & $0.78(0.27)$ \\
$\quad$ Tremor & Wrist & $0.71(0.35)$ & $0.70(0.35)$ \\
$\quad$ Nontremor & Wrist & $0.79(0.31)$ & $0.81(0.21)$ \\
Percentage moving $\left(\% V_{\theta}\right)$ & & & \\
All patients & Leg & $0.78(0.34)$ & $0.82(0.27)$ \\
Tremor & Trunk & $0.62(0.39)$ & $0.81(0.34)$ \\
$\quad$ Nontremor & Leg & $0.89(0.14)$ & $0.89(0.16)$ \\
Percentage of dominant & & & \\
$\quad$ frequencies $\left(\% \mathrm{PF}_{4}\right)$ & & & \\
All patients & Trunk & $0.96(0.11)$ & $0.95(0.12)$ \\
Tremor & Leg & $0.97(0.07)$ & $0.97(0.05)$ \\
$\quad$ Nontremor & Trunk & $0.97(0.08)$ & $0.96(0.06)$ \\
\hline
\end{tabular}

tremor group than for the tremor group for the leg $(P<$ $0.05)$.

To compare our results with the study by Hoff and colleagues, ${ }^{21}$ the mean accelerometer signal in the frequency range between 1 and $3.5 \mathrm{~Hz}$ for 1-minute intervals $\left(\overline{\mathrm{a}}_{1-35}\right)$ was calculated just as in the study by Hoff associates ${ }^{21}$ The variable $\overline{\mathrm{a}}_{1-35}$ of the wrist showed a sensitivity of 0.77 and specificity of 0.75 for all patients, which is in agreement with results of Hoff and coworkers. ${ }^{21}$

A neural network with two hidden units and four input parameters gave a sensitivity and specificity of 1.00 $(\mathrm{SD}=0.00)$ and $0.98(\mathrm{SD}=0.07)$, respectively (performance of $0.98 ; \mathrm{SD}=0.03$ ). The most important variable seemed to be the variable $\% \mathrm{PF}_{4}$ of the trunk and this variable alone gave a performance of 0.96 . The second most important variable was the variable $\% \mathrm{PF}_{4}$ of the most affected leg, which gave an increase in performance of 0.02 . The other two variables $\overline{\mathrm{V}}_{\theta}$ of the less affected arm and $\overline{\mathrm{V}}$ of the trunk added another 0.01 to the performance.

Figure 3 shows an example of the performance of the variable $\% \mathrm{PF}_{4}$ of the trunk for a patient without a tremor. It shows the unfiltered (solid line) and the filtered (dashed line) prediction by the variable $\% \mathrm{PF}_{4}$ of the trunk. The filtered variable was used to detect on and off states. The patient in Figure 3 was monitored for 3 hours 20 minutes and switched twice between motor states. This patient showed a sensitivity and specificity of 1.0 and 0.98 , respectively. The $\% \mathrm{PF}_{4}$ variable clearly changed when the patient 


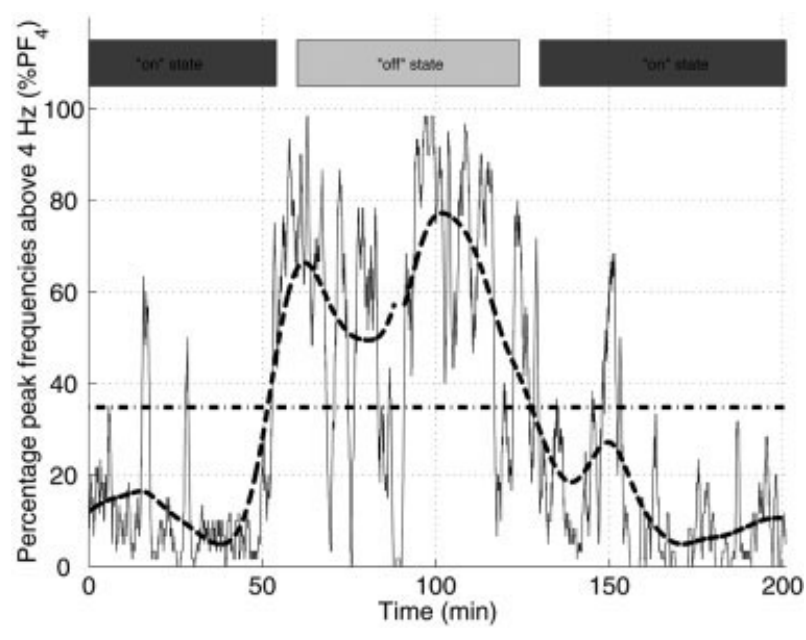

FIG. 3. Example of variable $\% \mathrm{PF}_{4}$ as a function of time for a nontremor patient who switched twice between motor states in a testing period of 3 hours and 20 minutes. The horizontal dashed-dotted line represents the threshold value of $34.8 \%$ to classify a patient as on (above the threshold) or as off(below the threshold). The solid line and the dashed line represent the unfiltered and filtered value of the variable $\% \mathrm{PF}_{4}$, respectively.

switched between motor states. This patient took his antiparkinson medication 210 minutes before the test and 70 minutes after the start of the test.

Each PD patient can be classified easily as a patient with a tremor or as a patient without a tremor. The tremor patients and nontremor patients showed different optimal classification thresholds (53.2\% and $34.8 \%$, respectively) and also seemed to have a different body segment (leg and trunk, respectively) that gave the best performance. Evaluating patients with a tremor or without a tremor in separate groups, each with their optimal classification threshold and body segment, gave a performance of 0.97 $(\mathrm{SD}=0.04)$, which was slightly better than the performance of 0.96 (SD = 0.09) when the patient group was evaluated as a whole. Only one patient showed a performance smaller than $0.90(0.89)$. This patient had a low sensitivity (0.78) and a high specificity (1.00). He did not suffer from a tremor and he mentioned that he was clearly off about 150 minutes after onset of the test. Figure 4 shows the prediction by the variable $\% \mathrm{PF}_{4}$ of the trunk for this patient. In the on state, the variable $\% \mathrm{PF}_{4}$ of the trunk was clearly below the threshold, which resulted in a specificity of 1.00. After about 140 minutes, the variable $\% \mathrm{PF}_{4}$ of the trunk showed a clear switch but the value was close to the optimal classification threshold of $34.8 \%$ in the off state. Because the variable $\% \mathrm{PF}_{4}$ was just below the optimal classification threshold for a small time period, this patient showed a relatively low sensitivity of 0.78 .

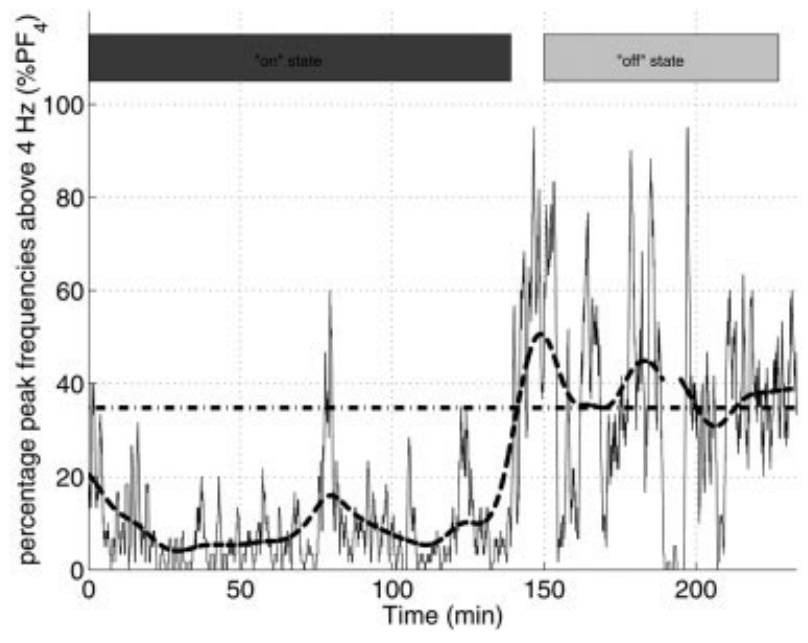

FIG. 4. Example of the percentage dominant frequencies $\left(\% \mathrm{PF}_{4}\right)$ for a nontremor patient who switched from the on state tot the off state after about 150 minutes. The horizontal dashed-dotted line represents the threshold value of $34.8 \%$ to classify a patient as on (above the threshold) or as off (below the threshold). The solid line and the dashed line represent the unfiltered and filtered value of the variable $\% \mathrm{PF}_{4}$, respectively. After about 190 minutes, the patient was walking for about 6 minutes; therefore, this part was excluded in the analysis.

For patients with a tremor, we expect most peak frequencies above $4 \mathrm{~Hz}$ in the off state. To illustrate differences in peak frequencies, we have calculated the distribution of the peak frequencies for the different motor states. Figure 5 shows a histogram of the peak frequencies for all patients in the on state (upper panel), for patients with a tremor in the off state (middle panel), and for patients without a tremor in the off state (lower panel). For PD patients in the on state, the peak of the
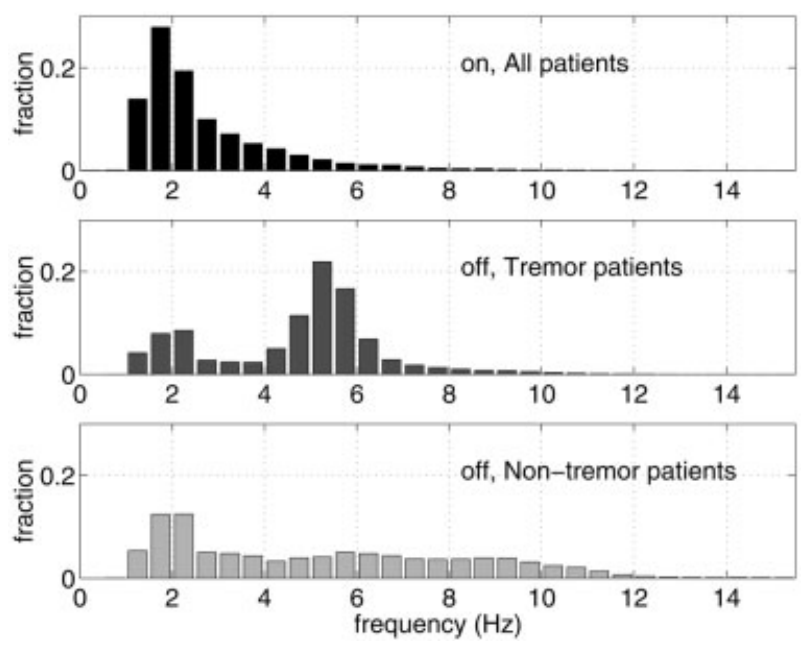

FIG. 5. Dominant frequencies for all patients in the on state (upper panel), patients with a tremor in the off state (middle panel), and patients without a tremor in the off state (lower panel). 


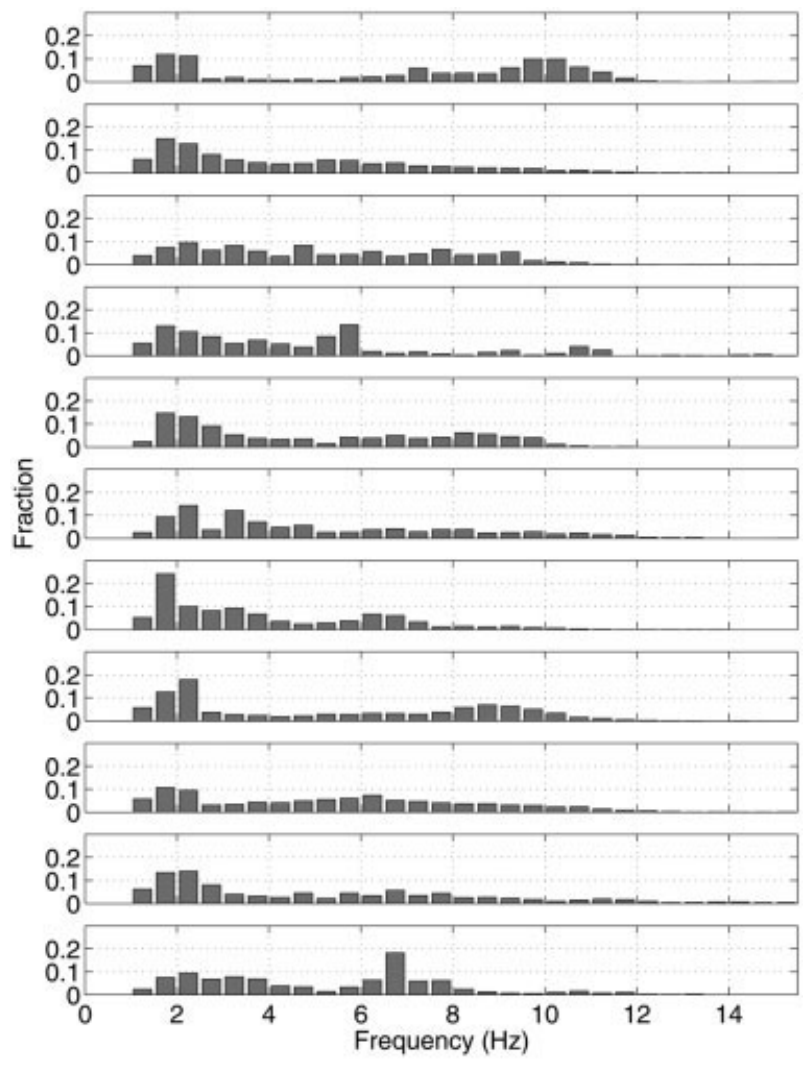

FIG. 6. Dominant frequencies for each nontremor patient in the off state.

histogram is mainly near $2 \mathrm{~Hz}$, with most frequencies below $4 \mathrm{~Hz}$ (upper panel of Fig. 5). A small peak in the histogram near $2 \mathrm{~Hz}$ is also seen for the PD patient groups with and without tremor in the off state (see middle and lower panel of Fig. 5). The middle panel of Figure 5 shows that patients with a tremor show most peak frequencies near $5 \mathrm{~Hz}$ in the off state, which was very consistent for all subjects. Quite surprisingly, PD Patients without a tremor showed also a large fraction of peak frequencies above $4 \mathrm{~Hz}$. The histogram has a broad distribution of peak frequencies with a relatively large fraction of 0.38 above $6 \mathrm{~Hz}$ (see lower panel of Fig. 5). These nontremor patients revealed large interindividual differences in the distribution of peak frequencies. The distributions for all patients without a tremor in the off phase are shown in Figure 6. The nontremor patients show a broad spectrum of dominant frequencies with different individual small peaks between 6 and $11 \mathrm{~Hz}$. In summary, PD patients with or without a tremor show a large number of dominant frequencies above $4 \mathrm{~Hz}$ in the off state, whereas the dominant frequencies are mainly around $2 \mathrm{~Hz}$ in the on state.
As mentioned earlier, one patient was left out of the data analysis because this patient was probably classified erroneously as on in a first rating for the whole testing period of 130 minutes. Reanalysis, however, demonstrated off symptoms in the legs (akathesia rather than LID), although the other parts of the body seemed to be in the on state. Because there remained some doubt about the nature of the dyskinesia of the legs, we decided to leave this patient out of the analysis. Interestingly, the variable $\% \mathrm{PF}_{4}$ was slightly above the detection threshold most of the time for this patient, which is consistent with a minor but significant rating as off.

\section{DISCUSSION}

Quantitative automatic assessment of the motor state of PD patients will be extremely helpful in optimizing individual medication and for evaluating new interventions in Parkinson's disease. Recently, several studies focused on the assessment of L-dopa induced dyskinesia in patients with Parkinson's disease. ${ }^{19,20,27,28}$ We used a neural network approach with good success to assess the severity of dyskinesia and to distinguish dyskinesia from voluntary movements in daily life. ${ }^{20}$ For practical purposes it is also important that such a method is able to assess parkinsonian symptoms, i.e., to distinguish between on and off states. So far, a successful automatic method to distinguish between on and off states has not been available. The main purpose of this study was to develop an automatic method to detect on and off states in daily life.

One of the most important core features of PD is bradykinesia. ${ }^{29}$ Timed motor tests and accelerometry demonstrated that PD patients move more slowly in the off state than in the on state and move more slowly than do age-matched controls. ${ }^{30,31}$ In a previous study, Hoff and colleagues ${ }^{21}$ used the mean acceleration of the arm as a measure for bradykinesia to assess on and off states. They found an average sensitivity of 0.66 ( 0.71 while upright and 0.61 while sitting) and an average specificity of 0.73 ( 0.76 while upright and 0.67 while sitting) for the mean acceleration of the arm. In our study, the same variable $\left(\bar{a}_{1-35}\right.$ of the wrist) revealed a sensitivity of 0.78 and a specificity of 0.75 , which was slightly better but roughly in agreement with the study of Hoff and coworkers $^{21}$ Our two variables for bradykinesia, $\overline{\mathrm{V}}$ and $\overline{\mathrm{V}}_{\theta}$, showed similar values for sensitivity $(0.73$ and 0.75 , respectively) and specificity (0.78 and 0.78 , respectively). These results show that PD patients move on average with a smaller velocity (i.e., bradykinetic) during the off state compared to that in the on state. Because the patients' motor behavior was not constrained by standardized execution of defined tasks, however, patients 
showed movements with a wide intra- and interindividual variety of velocities. This can result in smaller velocity values in the on state for fine movement tasks than for gross movement tasks such as washing the dishes in the off state. Furthermore, we also included the beginning of the end-of-dose periods, which is accompanied with less severe bradykinesia than that seen in the fully developed off period in most of our patients. As a result, the sensitivity and specificity for these bradykinesia related parameters are not good enough for practical use to distinguish between on and off states.

Another important core feature of PD is hypokinesia. Hoff and associates ${ }^{21}$ used the variable immobility periods as a measure for hypokinesia and found an average sensitivity of 0.65 ( 0.70 while upright and 0.60 while sitting) and an average specificity of 0.70 ( 0.73 while upright and 0.67 while sitting). This variable is similar to our variable "percentage moving" $\left(\% \mathrm{~V}_{\theta}\right)$. The variable $\% \mathrm{~V}_{\theta}$ gave a sensitivity of 0.79 and a specificity of 0.81 . The results of both studies indicate that PD patients are more hypokinetic in the off state than in the on state. The slightly higher performance in our study could be a result of several factors. Hoff and associates ${ }^{21}$ used self-assessment as a reference, whereas we used the assessment by an experienced neurologist as a reference. In addition, we excluded the time periods in which subjects were walking for more than 3 minutes. The reason for doing so is that patients are able to walk in the less severe off state; however, the variable $\% \mathrm{~V}_{\theta}$ will indicate continuous movement during walking. Hoff and colleagues ${ }^{21}$ therefore would classify patients as on when they are walking for a large part of the time in the off state, whereas we base our classification on the time when subjects are not walking. When walking induces problems in detecting the motor state in real daily life, spatiotemporal parameters of gait could be included to distinguish between on and off states as described by Salarian and coworkers. ${ }^{32}$ Whatever the reasons are for the small differences between the results in the study of Hoff and colleagues ${ }^{21}$ and our study, the sensitivity and specificity values for variables as percentage time of moving and immobility periods are too low for practical use in both studies to distinguish between on and off states.

An interesting finding for the variables $\overline{\mathrm{V}}$ and $\% \mathrm{~V}_{\theta}$ was the difference in performance between the nontremor and the tremor group. The nontremor group showed a significantly better performance than did the tremor group for hypokinetic $(\overline{\mathrm{V}})$ and bradykinetic $\left(\% \mathrm{~V}_{\theta}\right)$ parameters. This cannot be a result of tremor, because the accelerometer signals were filtered with a low-pass filter with a cut-off frequency at $3.5 \mathrm{~Hz}$ for the calculation of the hypokinetic and bradykinetic parame- ters. This finding supports the notion that rigid-akinetic PD patients mainly suffer from bradykinesia and hypokinesia and that tremor-dominant PD patients suffer less from bradykinesia and hypokinesia. ${ }^{33}$

The variable $\% \mathrm{PF}_{4}$ showed an excellent performance in detecting the on and off state. The variable $\% \mathrm{PF}_{4}$ of the trunk alone, gave a sensitivity and specificity of 0.96 and 0.95 for the PD patients group as a whole. For nontremor patients, the optimal classification threshold was $34.8 \%$ and the optimal segment was the trunk, whereas the tremor patients had the leg as optimal segment and an optimal classification threshold of $53.2 \%$. When both groups were analyzed separately, the sensitivity and specificity increased to 0.97 . Only 1 patient showed a performance smaller than 0.90 . The value of the variable $\% \mathrm{PF}_{4}$ of the trunk for this patient fluctuated near the optimal classification threshold and did not exceed the classification threshold at all times in the off state. Our neural network approach combined the variable $\% \mathrm{PF}_{4}$ of the trunk and the variable $\% \mathrm{PF}_{4}$ of the leg as the two most important parameters for the tremor and nontremor groups of patients, respectively. The neural networks approach gave a slightly better performance in detecting the motor state than did the variable $\% \mathrm{PF}_{4}$ alone.

The obvious question to ask is why do all PD patients, including the patients without a tremor in the off state, show a large percentage of peak frequencies above $4 \mathrm{~Hz}$ in the off state but not in the on state? In daily life, most movements appear at low frequencies, in particular below $1 \mathrm{~Hz}^{22}$ For the calculation of the variable $\% \mathrm{PF}_{4}$, we filtered the raw accelerometer signals with a high-pass filter with a cut-off frequency at $1 \mathrm{~Hz}$. The peak of the histogram near $2 \mathrm{~Hz}$ in the on and off state (see Fig. 4) therefore is most likely due to voluntary movements. Because patients in the on state make movements with normal range of frequencies and amplitudes, a large percentage of peak frequencies will be seen near $2 \mathrm{~Hz}$ and not above $4 \mathrm{~Hz}$. Moreover, dyskinetic movements are mainly in the frequency range below $3 \mathrm{~Hz} \cdot{ }^{19,27,34}$ Peak frequencies below $4 \mathrm{~Hz}$ therefore are also expected for patients with dyskinetic movements in the on state. This means that dyskinetic movements will help in classifying a patient as on. In our study, about half of the patients suffered from dyskinesia in the on state.

Many patients in the off state have a parkinsonian rest tremor with a typical frequency between 4 and $8 \mathrm{~Hz}^{23,24}$; therefore, patients with a tremor in the off state will show a large percentage of peak frequencies above $4 \mathrm{~Hz}$.

For patients without a tremor, we also found a relatively large fraction of peak frequencies above $4 \mathrm{~Hz}$ in the off state (see Figs. 5 and 6). Based on the results of this study, we can only speculate about the origin of these frequencies. The nontremor patients showed a 
broad spectrum of peak frequencies with various small peaks in the range between 6 and $11 \mathrm{~Hz}$ in the off state (see Fig. 6). The frequency of these peaks might be related to the postural tremor between 5 and $12 \mathrm{~Hz}$ reported in patients with PD. ${ }^{23,35-37}$ Postural tremor has also been reported for patients without clinical signs of tremor. ${ }^{38}$ The amplitudes of the dominant frequency peaks in our study were small and were best recorded in the trunk. They were found mainly when patients were at rest and when they abstained from voluntary movements. We believe that the detection of the high-frequency peaks was best for the trunk because the trunk is involved mainly in maintaining posture and less involved in voluntary movements, which typically are in the frequency range below $2 \mathrm{~Hz}$. Another reason why these highfrequency peaks might be due to a postural tremor and mainly detected in the off state is the difference in amplitude of the tremor in the on and off state. Recently, Sturman and colleagues ${ }^{39}$ showed that the amplitude of both resting and postural tremor decreased when patients were on compared with off. This suggests that rest and postural tremors are present in the on state but less prominent than in the off state. Based on our findings, we suggest that the large fraction of peak frequencies above $4 \mathrm{~Hz}$ in nontremor patients results from a postural tremor, which is best recorded in the off state. We do not have objective evidence, however, that the high-frequency peaks in nontremor patients are caused by a postural tremor. The high-frequency peaks could also be caused by physiological tremor reported in healthy subjects, which have a frequency in the range between 8 and $12 \mathrm{~Hz}^{37}$ Another explanation for the high-frequency peaks could be microvibrations of the body surface, which are in a frequency range of 7 to $13 \mathrm{~Hz} \cdot{ }^{40}$ Cardiac output and resting activity in skeletal muscle have been thought to be responsible for this phenomenon.

Irrespective of the origin of these high-frequency peaks, they were best recorded when patients abstained from any voluntary movements. In the off state, patients abstain more often from voluntary movements than they do in the on state and this condition will thus result in a higher probability of measuring high-frequency peaks. In addition, voluntary movements are more dominant and frequent in the on state and thus make it more difficult to detect small-amplitude high-frequency peaks in the on state. In conclusion, PD patients have their peak frequencies mainly above $4 \mathrm{~Hz}$ in the off state due to a rest or postural tremor in tremor patients, or due to postural tremor or another high-frequency movement in nontremor patients, whereas the peak frequencies are mainly below $4 \mathrm{~Hz}$ in the on state due to voluntary movements.
In conclusion, in an earlier study we were able to develop an algorithm to discriminate LID from voluntary movements. In the present study, we have developed the next step for automatic detection of daily performance in PD patients, namely an automatic, unsupervised algorithm that can distinguish between on and off states with a sensitivity and specificity near 0.97 . The method used in this study can automatically assess the motor state of PD patients and can operate successfully in unsupervised ambulatory conditions. The method requires a simple triaxial accelerometer placed on the most-affected leg for patients with a tremor and on the trunk for patients without a tremor.

Acknowledgments: This study was financially supported by the Prinses Beatrix Fonds (project-number: MAR 02-0219) and the Parkinson Patienten Vereniging. We thank the Department of Occupational Therapy of the University Medical Center St. Radboud for the opportunity to use their facilities for the measurements in this study.

\section{REFERENCES}

1. Fabbrini G, Juncos JL, Mouradian MM, Serrati C, Chase TN. Levodopa pharmacokinetic mechanisms and motor fluctuations in Parkinson's disease. Ann Neurol 1987;21:370-376.

2. Fabbrini G, Mouradian MM, Juncos JL, Schlegel J, Mohr E, Chase TN. Motor fluctuations in Parkinson's disease: central pathophysiological mechanisms, Part I. Ann Neurol 1988;24:366-371.

3. Horstink MW, Zijlmans JC, Pasman JW, Berger HJ, van 't Hof MA. Severity of Parkinson's disease in a risk factor for peak-dose dyskinesia. J Neurol Neurosurg Psychiatry 1990;53:224-226.

4. Nutt JG, Carter RN, Woodward WR. Long-duration response to levodopa. Neurology 1995;45:1613-1616.

5. Hoehn MM, Yahr MD. Parkinsonism: onset, progression and mortality. Neurology 1967;17:427-442.

6. Goetz CG, Stebbins GT, Shale HM, Lang AE, Chernik DA, Chmura TA, Ahlskog JE, Dorflinger EE. Utility of an objective dyskinesia rating scale for Parkinson's disease: inter- and intrarater reliability assessment. Mov Disord 1994;9:390-394.

7. Lang AE. Clinical rating scales and videotape analysis. In: Koller WC, Paulson G, editors. Therapy of Parkinson's disease. New York: Marcel Dekker; 1995. p 21-46.

8. Golbe LI, Pae J. Validity of a mailed epidemiological questionnaire and physical self-assessment in Parkinson's disease. Mov Disord 1988;3:245-254.

9. Brown RG, MacCarthy B, Jahanshahi M, Marsden CD. Accuracy of self-reported disability in patients with parkinsonism. Arch Neurol 1989;46:955-959.

10. Vitale C, Pellecchia, MT, Grossi D, Fragassi N, Cuomo T, Di Maio L, Barone P. Unawareness of dyskinesia in Parkinson's and Huntington's diseases. Neurol Sci 2001;22:105-106.

11. Goetz CG, Stebbins GT, Blasucci LM, Grobman MS. Efficacy of a patient-training videotape on motor fluctuations for on-off diaries in Parkinson's disease. Mov Disord 1997;12:1039-1041.

12. Montoye HJ, Washburn R, Servais S, Ertl A, Webster JG, Nagle FJ. Estimation of energy expenditure by a portable accelerometer. Med Sci Sports Exerc 1983;15:403-407

13. Bouten CV, Westerterp KR, Verduin M, Janssen JD. Assessment of energy expenditure for physical activity using triaxial accelerometer. Med Sci Sports Exerc 1994;26:1516-1523

14. Van Hilten JJ, Middelkoop HAM, Kerkhof GA, Roos RA. A new approach in the assessment of motor activity in Parkinson's disease. J Neurol Neurosurg Psychiatry 1991;54:976-979. 
15. Van Hilten JJ, Hoogland G, van der Velde EA, van Dijk JG, Kerkhof GA, Roos RA. Quantitative assessment of parkinsonian patients by continuous wrist activity monitoring. Clin Neuropharmacol 1993;16:36-45.

16. Van Hilten JJ, Kabel JF, Middelkoop HA, Kramer CG, Kerkhof GA, Roos RA. Assessment of response fluctuations in Parkinson's disease by ambulatory wrist activity monitor. Acta Neurol Scand 1993;87:171-177.

17. Katayama S. Actigraph analysis of diurnal motor fluctuations during dopamine agonsit therapy. Eur Neurol 2001;46:11-17.

18. Dunnewold RJ, Hoff JI, van Pelt HC, Fredrikze PQ, Wagemans EA, van Hilten BJ. Ambulatory quantitative assessment of body position, bradykinesia, and hypokinesia in parkinson's disease. J Clin Neurophysiol 1998;15:235-242.

19. Hoff JI, van de Plas AA, Wagemans EA, van Hilten JJ. Accelerometric assessment of levodopa-induced dyskinesias in Parkinson's disease. Mov Disord 2001;16:58-61.

20. Keijsers NL, Horstink MW, Gielen SC. Automatic assessment of levodopa induced dyskinesias in daily life by neural networks. Mov Disord 2003;8:70-80.

21. Hoff JI, van der Meer V, van Hilten JJ. Accuracy of objective ambulatory accelerometry in detecting motor complications in patients with Parkinson disease. Clin Neuropharmacol 2004;27: 53-57.

22. Howard I, Körding K, Ingram J, Wolpert D. Statistics of natural arm movements. Thirteenth annual meeting of neural control of movement. Sitges; 2004.

23. Elble RJ Jr, Koller WC. Tremor. Baltimore (MD): John Hopkins University Press; 1990.

24. Deuschl G, Raethien J, Baron R, Lindemann M, Wilms H, Krack P. The pathophysiology of parkinsonian tremor: a review. J Neurol 2000;247(Suppl.):33-48.

25. Bussmann JB, Damen L, Stam HJ. Analysis and decomposition of signals obtained by thigh-fixed accelerometry during walking. Med Biol Eng Comp 2000;38:632-638.

26. Zijlstra W, Hof AL. Assessment of spatio-temporal gait parameters from trunk accelerations during human walking. Gait Posture 2003;18:1-10.

27. Manson AJ, Brown P, O'Sullivan JD, Asselman P, Buckwell D, Lees AJ. An ambulatory dyskinesia monitor. J Neurol Neurosurg Psychiatry 2000;68:196-201.
28. Burkhard PR, Shale H, Langston W, Tetrud JW. Quantification of dyskinesia in parkinson's disease: validation of a novel instrumental method. Mov Disord 1999;14:

29. Marsden CD. The mysterious motor function of the basal ganglia: the Robert Wartenberg Lecture. Neurology 1982;32:514-539.

30. Dunnewold RJW, Jacobi CE, van Hilten JJ. Quantitative assessment of bradykinesia in patients with Parkinson's disease. J Neurosci Methods 1997;74:107-112.

31. Metman LV, Myre B, Verwey N, Hassin-Baer S, Arzbaecher J, Sierens D, Bakay R. Test-retest reliability of UPDRS-III, dyskinesia scales, and timed motor tests in patients with advanced Parkinson's disease: an argument against multiple baseline assessments. Mov Disord 2004;19:1079-1084.

32. Salarian A, Russmann H, Vingerhoets FJ, Dehollain C, Blanc Y, Burkhard PR, Aminian K. Gait assessment in Parkinson's disease: toward an ambulatory system for long-term monitoring. IEEE Trans Biomed Eng 2004;51:1434-1443.

33. Jankovic J, McDermott M, Carter J, Gauthier S, Goetz C, Golbe L, Huber S, Koller W, Olanow C, Shoulson I, et al. Variable expression of Parkinson's disease: a base-line analysis of the DATATOP cohort. The Parkinson Study Group. Neurology 1990;400:1529_ 1534.

34. Keijsers NL, Horstink MW, Gielen SC. Movement parameters that distinguish between voluntary movements and levodopa-induced dyskinesia in Parkinson's disease. Hum Mov Sci 2003;18:70-80.

35. Findley LJ, Gresty MA, Halmagyi GM. Tremor, the cogwheel phenomenon and clonus in Parkinson's disease. J Neurol Neurosurg Psychiatry 1981;44:534-546.

36. Deuschl G, Bain P, Brin M. Consensus statement of the movement disorder society on tremor. Ad Hoc Scientific Committee. Mov Disord 1998;13(Suppl.):2-23.

37. McAuley JH, Marsden CD. Physiological and pathological tremors and rhythmic central motor control. Brain 2000;123:1545-1567.

38. Vaillancourt DE, Newell KM. The dynamics of resting and postural tremor in Parkinson's disease. Clin Neurophysiol 2000;111: $2046-2056$.

39. Sturman MM, Vaillancourt DE, Metman LV, Bakay RA, Corcos DM. Effects of subthalamic nucleus stimulation and medication on resting and postural tremor in Parkinson's disease. Brain 2004; 127:2131-2143.

40. Gallasch E, Kenner T. Characterisation of arm microvibration recorded on an accelerometer. Eur J Appl Physiol Occup Physiol 1997;75:226-232. 